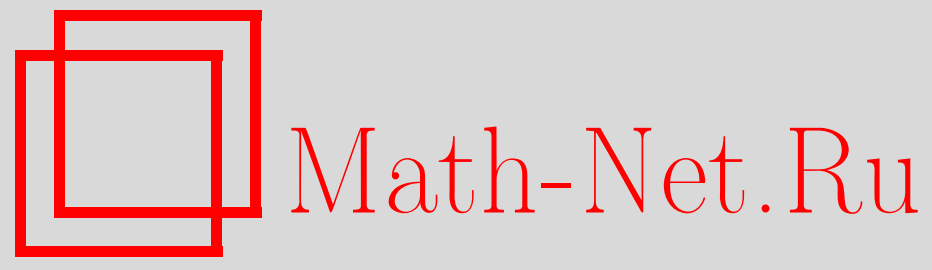

Д. Е. Кислов, А. Н. Прилуцкий, Критерий пространственно-возрастной организации древостоя, Вестн. Сам. гос. техн. ун-та. Сер. Физ.-мат. науки, 2012, выпуск 1(), 175-182

DOI: https://doi.org/10.14498/vsgtu955

Использование Общероссийского математического портала Math-Net.Ru подразумевает, что вы прочитали и согласны с пользовательским соглашением

http://www.mathnet.ru/rus/agreement

Параметры загрузки:

IP : 44.207 .124 .84

26 апреля 2023 г., $17: 11: 28$

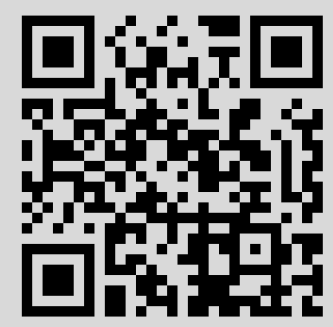


Вестн. Сам. гос. техн. ун-та. Сер. Физ.-мат. науки. 2012. № 1 (26). С. $175-182$

УДК 57:51-76; 57.02:001.57

\title{
КРИТЕРИЙ ПРОСТРАНСТВЕННО-ВОЗРАСТНОЙ ОРГАНИЗАЦИИ ДРЕВОСТОЯ
}

\author{
Д.Е. Кислов, А.Н. Прилуцкий \\ Ботанический сад-институт ДВО РАН, \\ Россия, 690024, Владивосток, ул. Маковского, 142. \\ E-mails: kisl_di@mail.ru, prilutsky@bgi.dvo.ru
}

\begin{abstract}
Рассматривается статистический критерий для проверки однородности пространственного распределения деревъев в лесном сообществе с учётом его возрастного состава. Даётся физическая интерпретация статистики критерия и приводятся резулътаты вычислительных экспериментов, выполненных на натурных данных.
\end{abstract}

Ключевые слова: динамика растительного покрова, пространственная организачия древостоя, критерий случайности.

Введение. Одним из традиционных средств статистического анализа особенностей размещения деревьев в лесном сообществе является анализ значений специальных функций, предложенных B. Ripley $[1,2]$.

Начиная с пионерских работ в этой области $[3,4]$ и заканчивая последними модификациями подхода B. Ripley [5,6] следует отметить определенный односторонний характер предлагаемых критериев, направленных на установление либо разреженности, либо избыточного скопления объектов в пространстве.

В настоящей работе предлагается принципиально другой статистический подход к изучению горизонтальной структуры древесных сообществ, позволяющий локально характеризовать тенденции их пространственно-временного развития. Подход заключается в последовательном анализе размещения объектов с учётом их возраста в каждой подобласти заданного участка местности в свете представлений о волновом характере пространственно-временной динамики популяций [7]. Статистический критерий для проверки случайности пространственно-возрастного распределения объектов строится на базе вводимых числовых характеристик выраженности волнового процесса.

Формулировка метода. Как и в [1,2], ограничимся локальным анализом при исследовании особенностей совместного размещения объектов в пространстве.

Пусть $W_{r}(\mathbf{y})$ - круговое окно известного радиуса $(r)$ с координатами центра у. Характеризуем положение в пространстве и возраст объекта (дерева) парой случайных величин $\mathbf{X}$ (здесь и далее по тексту жирным шрифтом выделены вектора или матрицы, причём полагается, что вектора - векторстолбцы) и $A$ соответственно, имеющих плотность совместного распределения $u(\mathbf{X}, A)$, равную нулю вне окна $W_{r}(\mathbf{y})$ и удовлетворяющую условию нормировки. Заметим, что поскольку в работе рассматриваются вопросы, связанные исключительно с горизонтальным размещением объектов, случайная величина $\mathbf{X}$ полагается двумерной.

Идея предлагаемого статистического подхода заключается в отыскании

Дмитрий Евгенъевич Кислов (к.ф.-м.н.), научный сотрудник, лаб. экологии растительного покрова. Александр Николаевич Прилуцкий (к.с.-х.н.), ведущий научный сотрудник, лаб. экологии растительного покрова. 
единичного направления $\mathbf{n}$, доставляющего экстремальные значения коэффициенту корреляции $\rho\left(\mathbf{n}^{\top} \mathbf{X}, A\right)$ и проходящего через центр данного окна.

Из постановки оптимизационной задачи и свойств коэффициента корреляции следует, что выбор $\mathbf{n}$, по крайней мере, двузначен и определяется с точностью до направления: если $\mathbf{n}$ доставляет экстремальное (например, максимальное) значение $\rho\left(\mathbf{n}^{\top} \mathbf{X}, A\right)$, то и $-\mathbf{n}$ также будет доставлять этой величине экстремальное (но уже минимальное) значение. Разрешение этой неоднозначности обеспечивается выбором такого направления, которое допускает более ценную интерпретацию.

В качестве основы для такой интерпретации в работе принято явление распространения волн плотности ценопопуляций $[7,8]$. Если направление $\mathbf{n}$ выбрано так, что оно указывает на скопление деревьев с малым возрастом, то оказывается возможным локально отождествить $\mathbf{n}$ с фронтом распространения волны.

Проведем рассуждения, рассматривая для определенности три возрастных группы деревьев, условно: младшую, среднюю и старшую. Ограничиваясь только старшей возрастной группой и экстраполируя процесс дальнейшего развития сообщества, можно представить вероятную (локальную) тенденцию пространственного изменения плотности распределения деревьев этой возрастной группы: те экземпляры, которые в настоящий момент являются наиболее старшими, через некоторое время выпадут, и в эту (старшую) возрастную группу попадут деревья, которые в настоящий момент относятся к средней возрастной группе; аналогично, в среднюю возрастную группу попадут представители младшей возрастной группы; таким образом, плотность распределения деревьев старших возрастных групп имеет тенденцию смещаться в направлении, задаваемом $\mathbf{n}$.

Рассмотрим практические вопросы реализации предложенного подхода и сформулируем на его основе статистический критерий.

Сохраняя введённые выше обозначения, придём к необходимости оптимизации некоторой оценки коэффициента корреляции $\hat{\rho}\left(\mathbf{n}^{\top} \hat{\mathbf{X}}, \hat{\mathbf{A}}\right)$ по выборочным данным $(\hat{\mathbf{X}}, \hat{\mathbf{A}})$, где $\hat{\mathbf{X}}$ - выборка векторов координат объектов, $\hat{\mathbf{A}}-$ соответствующий набор оценок их возрастов. В контексте работы полагается, что $\hat{\mathbf{X}}=\left(\hat{\mathbf{X}}_{1}, \hat{\mathbf{X}}_{2}, \ldots, \hat{\mathbf{X}}_{N}\right)$, где $\mathbf{X}_{i}$ - вектор координат $i$-того объекта, $i=1,2, \ldots, N, N$ - общее число объектов, $\hat{\mathbf{A}}=\left(\hat{A}_{1}, \hat{A}_{2}, \ldots, \hat{A}_{N}\right)$, а символом «крышечка» обозначены выборочные данные. Таким образом, $\operatorname{dim} \hat{\mathbf{X}}=2 \times N$ и произведения вида $\mathbf{n}^{\top} \hat{\mathbf{X}}$ корректно определены в соответствии с традиционными представлениями.

Пусть $\mathrm{E}(\cdot)$ и $\mathrm{D}(\cdot)=\mathrm{E}((\cdot)-\mathrm{E}(\cdot))^{2}$ - операторы взятия выборочных оценок математического ожидания и дисперсии соответственно. Для определённости положим $\mathrm{E} \hat{\mathbf{X}}=N^{-1} \sum_{k=1}^{N} \hat{\mathbf{X}}_{k}$. Тогда в качестве оценки коэффициента корреляции может быть выбрано следующее [9]:

$$
\hat{\rho}\left(\mathbf{n}^{\top} \hat{\mathbf{X}}, \hat{\mathbf{A}}\right)=\frac{\mathbf{n}^{\top} \mathbf{Q}}{\sqrt{\mathbf{n}^{\top} \mathbf{K n}}}
$$

где

$$
\mathbf{Q}=\frac{1}{N \sqrt{\mathrm{D} \mathbf{A}}} \sum_{i=1}^{N}\left(\hat{\mathbf{X}}_{i}-\mathrm{E} \hat{\mathbf{X}}\right) \cdot\left(\hat{A}_{i}-\mathrm{E} \hat{\mathbf{A}}\right), \quad \mathbf{K}=\frac{1}{N} \sum_{i=1}^{N}\left(\hat{\mathbf{X}}_{i}-\mathrm{E} \hat{\mathbf{X}}\right)\left(\hat{\mathbf{X}}_{i}-\mathbf{E} \hat{\mathbf{X}}\right)^{\top}
$$


Заметим, что для дальнейшего изложения важна форма выражения (1), при этом в качестве $\mathbf{Q}$ и $\mathbf{K}$ могут использоваться различные статистические оценки; это не повлияет на приводимые далее вычислительные аспекты нахождения оптимального направления $\mathbf{n}$.

Выясним условия существования направления, доставляющего экстремальное значение выражению $\hat{\rho}$ в $(1)$.

Конечность величины $\hat{\rho}$ обеспечивается требованием D $\hat{\mathbf{A}}>0$, означающим, что в текущем зондирующем окне $W(\mathbf{y})$ находится, по крайней мере, два объекта различного возраста, и положительной определенностью оператора автокорреляции $\mathbf{K}$, и также является не обременительным на практике ограничением.

Определим экстремальные направления для $\hat{\rho}$ при условии, что $\|\mathbf{n}\|=1$. Полагая $\mathbf{n}=(\cos (\varphi), \sin (\varphi))^{\top}$, выпишем необходимые условия экстремума функции $\hat{\rho}$ :

$$
\begin{aligned}
\frac{d \hat{\rho}\left(\mathbf{n}^{\top} \hat{\mathbf{X}}, \hat{\mathbf{A}}\right)}{d \varphi}=-\frac{1}{\left(\mathbf{n}^{\top} \mathbf{K n}\right)^{3 / 2}}(\sin (\varphi) & Q_{1} K_{22}-\sin (\varphi) Q_{2} K_{12}+ \\
& \left.+\cos (\varphi) Q_{1} K_{12}-\cos (\varphi) Q_{2} K_{11}\right)=0
\end{aligned}
$$

где $Q_{k}, K_{i j}$ - элементы матриц $\mathbf{Q}$ и $\mathbf{K}$, определённых в (1). Поскольку знаменатель в уравнении (2) в силу положительной определённости матрицы $\mathbf{K}$ положителен, нетрудно выписать решения этого уравнения в явном виде:

$$
z_{k}=\operatorname{arctg} \frac{Q_{2} K_{11}-Q_{1} K_{12}}{Q_{1} K_{22}-Q_{2} K_{12}}+\pi k, \quad k \in \mathbb{Z} .
$$

Учитывая $2 \pi$-периодичность функции $\hat{\rho}(\varphi)$, для определения $\mathbf{n}$ достаточно ограничиться одним значением $\varphi_{0}=z_{0}$. Проделав дополнительные вычисления, можно показать, что $d^{2} \hat{\rho} / d \varphi^{2} \neq 0$ при $\varphi=\varphi_{0}$. Узнать, является ли найденное экстремальное положение точкой максимума или минимума $\hat{\rho}$, можно из выражения (1). При $\mathbf{n}\left(\varphi_{0}\right)^{\top} \mathbf{Q}>0$ направление $\mathbf{n}\left(\varphi_{0}\right)$ доставляет максимум $\hat{\rho}$, при $\mathbf{n}\left(\varphi_{0}\right)^{\top} \mathbf{Q}<0-$ минимум, при $\mathbf{n}\left(\varphi_{0}\right)^{\top} \mathbf{Q}=0$ (т. е. $\mathbf{Q}=\mathbf{0}$ ) имеет место случай отсутствия корреляции между возрастом и проекциями координат положения объектов внутри зондирующего окна при любом возможном направлении $\mathbf{n}$. Последняя ситуация крайне маловероятна на практике и может быть исключена, например, введением дополнительного ограничения на минимальное по модулю допустимое значение $\hat{\rho}$.

Важно отметить, что вид функции, количественно отражающей зависимость между проекциями координат объектов и их возрастом (или какимилибо другими характеристиками), может выбираться весьма произвольно; в частности, специального внимания заслуживают функции, построенные на базе ранговых статистик и позволяющие прийти к не менее ценным интерпретациям.

Рассмотрим вопрос об исследовании случайности пространственного размещения деревьев. Традиционный подход к решению задачи заключается в её представлении в свете аппарата теории случайных процессов [1-3]. При этом в качестве критерия устанавливается скопление или разреженность размещения объектов в пространстве. В качестве модели случайного пространственно-возрастного распределения объектов примем независимое равномерное распределение $N$ объектов по площади и возрасту. 
На базе введённых представлений может быть построен статистический критерий случайности пространственного распределения объектов, интерпретация которого существенно отлична от приводимой в упомянутых выше работах и связана с обозначенным волновым характером пространственновременной динамики ценопопуляции. Сформулируем один из возможных вариантов такого статистического критерия.

Идеализированное представление о случайности характеризуется набором независимых равномерно распределённых случайных величин $\left\{\left(\mathbf{X}_{1}, A_{1}\right)\right.$, $\left.\left(\mathbf{X}_{2}, A_{2}\right), \ldots,\left(\mathbf{X}_{N}, A_{N}\right)\right\}$, где $\mathbf{X}_{i}$ - вектор пространственных координат, а $A_{i}-$ возраст объекта. Обозначим через $M$ количество случайно выбираемых на исследуемой площади зондирующих окон радиуса $r$. На основе экстремальных значений коэффициента корреляции и направлений $\mathbf{n}$ может быть построена статистика $\left.Z_{*}\left(M, \rho_{n}^{j}, \mathbf{n}_{j}, r\right\}\right)$, где $j=1,2, \ldots, M$ (конкретный вид используемых статистик приводится ниже). Число зондирующих окон $M$ определяет чувствительность критерия. Критическая область для принятия решения определяется по результатам статистического моделирования (метод МонтеКарло) для каждого значения $r$.

Определив критическую область $I(r, \alpha)=\left[Z_{*}^{-}(r, \alpha), Z_{*}^{+}(r, \alpha)\right]$ при некотором уровне значимости $\alpha$, необходимо проверить принадлежность вычисленного значения статистики найденному интервалу. Гипотеза о случайном характере пространственно-возрастной структуры не отвергается при данном уровне значимости $\alpha$, если значение статистики оказывается внутри интервала $I(r, \alpha)$, и отвергается в противном случае. На практике представляется более удобным графическое (по аналогии с работами $[2,5])$ изображение $Z_{*}(r)$ и соответствующих граничных значений $I(r, \alpha)$ в зависимости от $r$.

Вычислительный эксперимент. Использование предложенного метода проиллюстрируем на примере обработки данных, полученных с пробной площади, заложенной в лесах Горнотаёжной станции ДВО РАН. Объектом исследования избрано многовидовое сообщество растений, автохтонно развивающееся на платообразной межгорной седловине после сплошной рубки. Микрорельеф участка пробной площади формируют слабо выраженные, беспорядочно расположенные возвышения и понижения, а общий уклон поверхности, ориентированный на северо-запад, не превышает $5^{\circ}$.

Применение метода предполагает наличие оцифрованных данных, содержащих локальные (или географические) координаты объектов и их возраст. Поскольку на момент написания статьи необходимые данные для получения таких оценок отсутствовали, требуемый для работы метода возраст деревьев был отождествлен с диаметром их стволов; учитывая принципиальную сторону настоящей работы, такое допущение является вполне правомерным.

Рассмотрим вопрос об исследовании пространственно-возрастной структуры сложения древостоя на примере дуба монгольского (Quercus mongolica Fisch. ex Ledeb.). В качестве статистик выберем

$$
Z_{\text {mean }}(r)=M^{-1} \sum_{i=1}^{M} \rho_{n}^{i}(r), Z_{\text {min }}(r)=\min _{i=1,2, \ldots, M} \rho_{n}^{i}(r), Z_{\text {ang }}(r)=M^{-1} \sum_{i=1}^{M} \angle \mathbf{n}^{i}(r),
$$

где $M$ - количество случайно размещенных зондирующих окон (опыт вычислений показал, что достаточно ограничиться $M=1000) ; \angle \mathbf{n}^{i}(r)-$ угол, 
определяющий оптимальное направление $\mathbf{n}$ в $i$-том зондирующем окне радиуса $r$ (в данной работе принималось, что $\angle \mathbf{n} \in[-\pi / 2,3 \pi / 2])$.

Заметим, что выбор статистик, вообще говоря, произволен, однако предложенные имеют вполне ясные интерпретации. Величина $Z_{\text {mean }}(r)$ характеризует выраженность пространственно-возрастной упорядоченности в среднем по площади; $Z_{\min }(r)$ определяет наиболее выраженную локальную упорядоченность древостоя; $Z_{\text {ang }}(r)$ характеризует среднее оптимальное направление по площади.

При построении критической области критерия с помощью метода Монте-Карло число статистических испытаний принималось равным 1000.

На рис. 1, 2 представлены результаты вычисления статистик $Z_{*}(r)$ для дуба монгольского и соответствующие им критические области. С учётом того, что данный вид имеет наиболее рассеянный по площади характер распределения, анализ упорядоченности его размещения весьма интересен.

Выход сплошной линии из области, задаваемой пунктирными линиями (критической области критерия), означает, что на данном масштабе расстояний $(r)$ гипотеза о случайном характере пространственно-возрастной структуры должна быть отвергнута.

Таким образом, из рис. 1 следует, что в среднем пространственно-возрастная организация дуба монгольского не должна рассматриваться как случайная на круговых участках с радиусом до 10 м. При этом для участков размером около 10 м на исследуемой площади наблюдается наибольшая упорядоченность в сложении древостоя. При больших радиусах зондирующих окон наблюдаемое пространственно-возрастное размещение объектов при данном уровне значимости не отличается от случайного. При $r=5 \div 10$ м на рис. 2 наблюдается существенное уклонение среднего угла оптимального направления, что также указывает на особенности организации дуба монгольского на данных пространственных масштабах.

В дополнение к рассмотренным статистикам исследовалась возможность

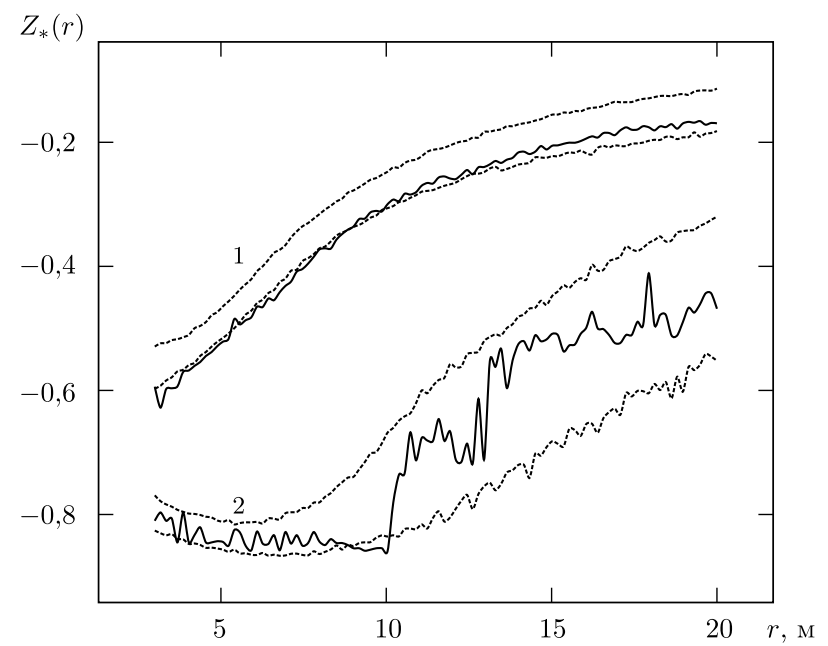

Рис. 1. Пространственно-возрастная организация дуба монгольского $(M=1000): 1-$ $Z_{*}(r)=Z_{\text {mean }}(r), 2-Z_{*}(r)=Z_{\min }(r)$, сплошная линия-значение статистики, пунктирная линия - критическая область критерия при $\alpha=0,05$ 


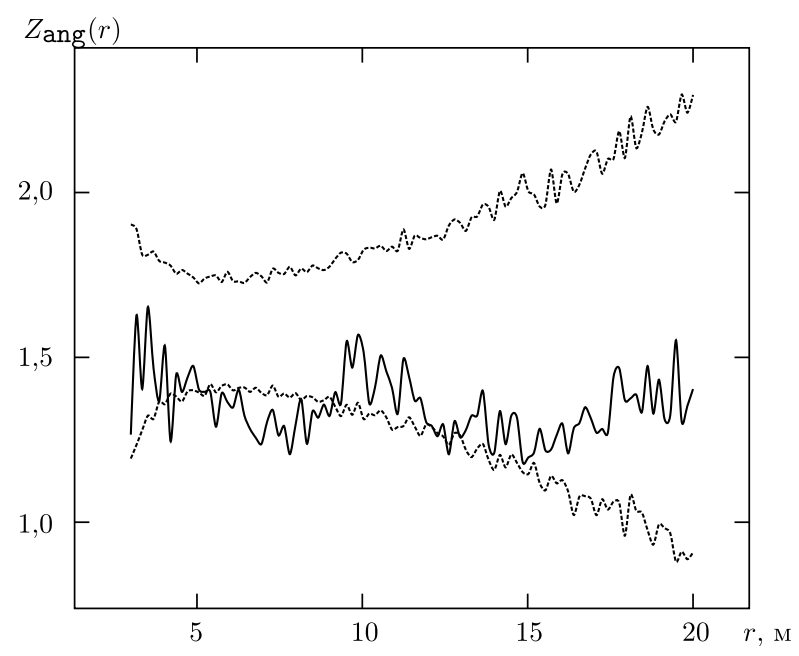

Рис. 2. Пространственно-возрастная организация дуба монгольского $(M=1000)$ : сплошная линия - значение статистики, пунктирная линия - критическая область критерия при $\alpha=0,05$

использования статистик, построенных на базе медианы, коэффициентов асимметрии и эксцесса. В результате вычислительных экспериментов было установлено, что данные показатели также чувствительны к особенностям пространственно-возрастного размещения объектов, однако наиболее чувствительной из них является статистика, построенная на базе медианы.

Учитывая принцип построения статистик, заметим, что они связаны с явлением распространения волн плотности в пространственно-временных моделях динамики популяций $[7,8]$. Например, для статистики $Z_{\min }(r)$ при $r=10$ м, вычисленной для дуба монгольского, могут быть построены оценки плотности вероятности присутствия объектов различных возрастных групп в проекции на оптимальное направление $\mathbf{n}$ (рис. 3).

На рис. 3 представлены оценки плотности распределения Парзена-Розенблатта [10] (при построении оценок использовалось гауссово ядро) для представителей трех возрастных групп дуба монгольского, находящихся в зондирующем окне с минимальным значением коэффициента корреляции (оценка плотности получена для проекций центров объектов на найденное оптимальное направление $\mathbf{n}$ ). Разбиение на три возрастные группы проводилось по диаметру стволов деревьев: интервал возможных диаметров образцов, попавших в окно, был разбит на три равные части; в соответствии с разбиением формировались три группы - старшая, средняя и младшая. Сплошная линия на рис. 3 - плотность распределения деревьев старшей возрастной группы, пунктирная - средней, точечная - младшей. Таким образом, представители старшей возрастной группы имеют тенденцию «смещаться» (локально!) в направлении n. Волновая интерпретация сохраняет свою силу и при больших размерах зондирующих окон, однако в этом случае пространственновозрастная упорядоченность объектов характеризуется меньшей величиной коэффициента корреляции.

Полученный «снимок» пространственной динамики может быть полезен при построении эвристических прогнозов развития растительного сообще- 


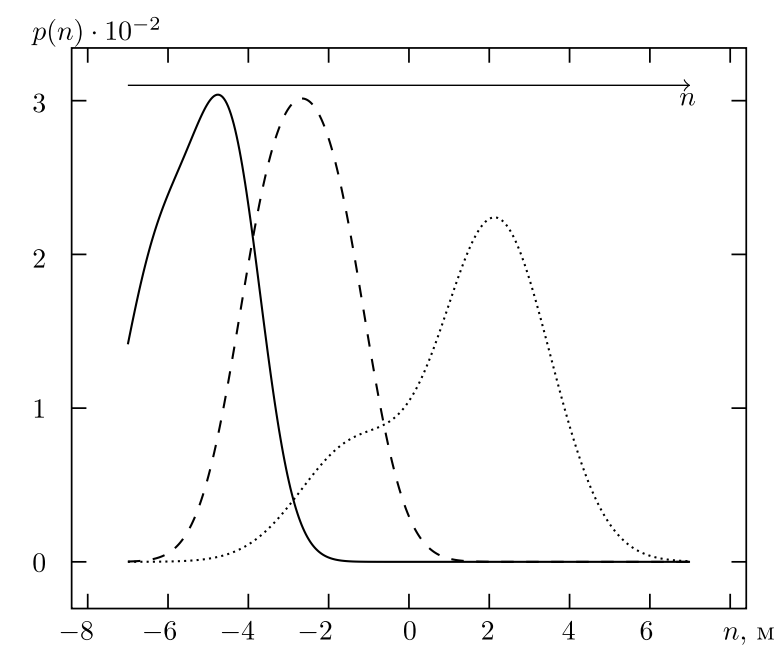

Рис. 3. Волны плотности в зондирующем окне

ства, анализа неоднородности его распределения по ареалу, изучении (идентификации) пространственных градиентов экологических факторов среды и исследовании особенностей совместного развития представителей видов в многовидовом сообществе.

В заключение перечислим возможные области применения предложенного метода (развитие которых требует отдельных исследований с учётом изложенных представлений):

- разработка, верификация и адаптация моделей пространственно-временной динамики биосистем;

- количественная и качественная характеристика динамики компонентов биосистем, сравнительный анализ их развития в различных природных условиях;

- исследование особенностей распределения абиотических и биотических факторов в экосистемах локального уровня;

- анализ пространственно-возрастной организации природных объектов.

\section{БИБЛИОГРАФИЧЕСКИЙ СПИСОК}

1. Ripley B.D. Modelling spatial patterns. With discussion // J. Roy. Statist. Soc. Ser. B, 1977. Vol. 39, no. 2. Pp. 172-212.

2. Ripley B. D. Tests of 'randomness' for spatial point patterns // J. Roy. Statist. Soc. Ser. B, 1979. Vol. 41. Pp. 368-374.

3. Clark P. J., Evans F. C. Distance to nearest neighbor as a measure of spatial relationships in populations // Ecology, 1954. Vol. 35, no. 4. Pp. 445-453.

4. Ripley B. D. The Second-Order Analysis of Stationary Point Processes // J. Appl. Probability, 1976. Vol. 13, no. 2. Pp. 255-266.

5. Diggle P. J. Statistical analysis of spatial point patterns / Mathematics in Biology. Vol. IX. London, New York etc.: Academic Press, 1983. 148 pp.

6. Marcon E., Puech F. Measures of the geographic concentration of industries: improving distance-based methods // J. Econ. Geogr., 2009. T. 10, № 5. С. 745-762.

7. Колмогоров А.Н., Петровский И. Г., Пискунов Н.С. Исследование уравнения диффузии, соединенной с возрастанием количества вещества и его применение к одной 
биологической проблеме // Бюл. МГУ. Матем. и механ., 1937. Т. 1, №6. С. 1-26; англ. пер.: Kolmogorov A., Petrovskii I., Piscounov N. A study of the diffusion equation with increase in the amount of substance, and its application to a biological problem / In: Selected Works of A. N. Kolmogorov. Vol. I; ed. V. M. Tikhomirov. Amsterdam: Kluwer Academic Publishers, 1991. Pp. 248-270.

8. Свирежев Ю. М. Нелинейные волны, диссипативные структуры и катастрофы в экологии.. М.: Наука, 1987. 367 с. [Svirezhev Yu. M. Nonlinear waves, dissipative structures and catastrophes in ecology. Moscow: Nauka, 1987. 367 pp.]

9. Кобзарь А.И. Прикладная математическая статистика для инженеров и научных работников. М.: Физматлит, 2003. 853 с. [Kobzar' A. I. Applied Mathematical Statistics for engineers and scientists. Moscow: Fizmatlit, 2003. 853 pp.]

10. Härdle W., Simar L. Applied multivariate statistical analysis. Berlin: Springer Verlag, 2003. $486 \mathrm{pp}$.

Поступила в редакцию $05 / \mathrm{IV} / 2011$;

в окончательном варианте - 19/II/2012.

\section{MSC: 93A30}

\section{THE CRITERION FOR SPACE-AGE ORGANIZATION OF STAND}

\section{E. Kislov, A. N. Prilutsky}

Botanical Garden-Institute FEB RAS, 142, Makovskogo st., Vladivostok, 690024, Russia.

E-mails: kisl_di@mail.ru, prilutsky@bgi.dvo.ru

A statistical criterion for randomness of spatial tree patterns in stands with account of trees age is proposed. Interpretation of the criterion statistic and numerical experiments on collected spatial data are presented.

Key words: vegetation cover dynamics, spatial organization of stand, randomness criterion.

Original article submitted 05/IV/2011; revision submitted 19/II/2012.

Dmitry E. Kislov (Ph. D. (Phys. \& Math.)), Research Scientist, Lab. of Vegetation Cover Ecology. Alexander N. Prilutsky (Ph. D. (Agric.)), Leading Research Scientist, Lab. of Vegetation Cover Ecology. 